\section{ScienceDirect}

Transportation Research Procedia 23 (2017) 720-737
Transportation

Research

Procedia

www.elsevier.com/locate/procedia

22nd International Symposium on Transportation and Traffic Theory

\title{
Stability analysis on a dynamical model of route choice in a connected vehicle environment
}

\author{
Seungjae Lee ${ }^{\mathrm{a}}$, Benjamin G. Heydecker ${ }^{\mathrm{b}}$, Jooyoung Kim ${ }^{\mathrm{c}, *}$, Sangung Park ${ }^{\mathrm{d}}$ \\ ${ }^{a}$ University of Seoul, Room 523, 21 st hall, University of Seoul, 163 Seoulsiripdaero, Dongdaemun-gu, Seoul 02504, KOREA \\ ${ }^{b}$ University College London, Centre for Transport Studies, London WC1E 6BT, UNITED KINGDOM \\ ${ }^{c}$ University of Seoul, Room 533, 21 st hall, University of Seoul, 163 Seoulsiripdaero, Dongdaemun-gu, Seoul 02504, KOREA \\ ${ }^{d}$ University of Seoul, Room 533, 21 st hall, University of Seoul, 163 Seoulsiripdaero, Dongdaemun-gu, Seoul 02504, KOREA
}

\begin{abstract}
Research on connected vehicle environment has been growing rapidly to investigate the effects of real-time exchange of kinetic information between vehicles and road condition information from the infrastructure through radio communication technologies. A fully connected vehicle environment can substantially reduce the latency in response caused by human perception-reaction time with the prospect of improving both safety and comfort. This study presents a dynamical model of route choice under a connected vehicle environment. We analyze the stability of headways by perturbing various factors in the microscopic traffic flow model and traffic flow dynamics in the car-following model and dynamical model of route choice. The advantage of this approach is that it complements the macroscopic traffic assignment model of route choice with microscopic elements that represent the important features of connected vehicles. The gaps between cars can be decreased and stabilized even in the presence of perturbations caused by incidents. The reduction in gaps will be helpful to optimize the traffic flow dynamics more easily with safe and stable conditions. The results show that the dynamics under the connected vehicle environment have equilibria. The approach presented in this study will be helpful to identify the important properties of a connected vehicle environment and to evaluate its benefits.
\end{abstract}

(C) 2017 The Authors. Elsevier B.V. All rights reserved.

Peer-review under responsibility of the scientific committee of the 22nd International Symposium on Transportation and Traffic Theory.

Keywords: Dynamical model. Route choice, Lyapunov stability, String stability, V2X, Connected vehicle

\section{Introduction}

Normal traffic flows can suffer from unexpected congestion due to an abrupt speed reduction or an incident. In such cases, perturbations cause dynamic increases in subsequent travel times. Traffic engineers and researchers argued that a connected vehicle (CV) environment leads to conditions with stabilized traffic flows and more predictable travel times. The CV environment will enable other vehicles, RSUs (Road Side Units), the infrastructure, and driver devices to send and receive real-time traffic information. It also offers the prospect of reducing accidents caused by driver

\footnotetext{
${ }^{*}$ Corresponding author. Tel.: +82-2-6490-5661 ; fax: +82-2-6490-2823.

E-mail address: trafficplan@ naver.com
} 
errors and phantom traffic jams in the form of stop-and-go waves caused by perception-reaction lags and geometric design (Knorr, et al., 2012).

The CV environment includes several emerging technologies such as sensor, mapping, recognition/decision, and radio communication. Previous research on $\mathrm{CV}$ environment significantly improved the technologies in these related fields. Among these technologies, researchers have focused on vehicle control strategies, communication with other vehicles, vehicle management, and connection to other advanced technologies. Traffic engineers, however, focused on appropriately revised microscopic traffic flow or lane-changing models to reflect the CV environment (Jia and Ngoduy, 2016; Talebpour and Mahmassani, 2016). Researchers were also interested in the accuracy, privacy, and effectiveness of radio communication between vehicles or high occupancy vehicles (HOV) such as bus or truck as well as connection with traffic signal controller using V2I (Bauza and Gozalvez, 2013; Knorr, et al., 2012). Traffic engineers indicated that a CV environment provides detailed information about driving conditions and can also improve road efficiency and safety. As a result, saturation flow can increase and traffic congestion be reduced.

In this study, we consider a microscopic traffic flow model to evaluate network stability because it is difficult to depict connected vehicles behavior in a macroscopic dynamical model of route choice due to the limitation of lowresolution data. Unlike the microscopic traffic flow model, the conventional macroscopic dynamical model of route choice will not explain the effect of real connected vehicles. Thus, a microscopic car-following model is used to depict $\mathrm{CV}$ environment accurately. For each time step, car-following models calculate the speed of a vehicle to maintain a relative gap between each vehicle and the preceding one. Gipps model and Intelligent Driver Model (IDM) are examples of car-following models (Treiber and Kesting, 2013). In the CV environment, the reaction time and hence safe gap can be decreases progressively by using Adaptive Cruise Control (ACC) and then Cooperative Adaptive Cruise Control (CACC) technologies.

Recently, Mahmassani (2016) has considered network performance under the CV environment. Although this plays an important role in traffic flowflow prediction, only one paper considered route choice process in the CV environment. Correia and Arem (2016) integrated traffic assignment and the parking strategy process considering automated vehicles into the user optimum privately owned automated vehicles assignment problem (UO-POAVAP). To measure the effectiveness of CV environment and reduce travel times under such road conditions require the study of traffic assignment considering $\mathrm{CV}$ environment.

The conventional mesoscopic traffic assignment process does not usually represent signals or intersections in detail. Previously, researchers just understood traffic assignment as a problem of finding the equilibrium pattern over a given urban transportation network (Sheffi, 1985). Smith (1979), Allsop (1974), and Bentley and Lambe (1980) were the first to combine models of route choice and traffic signal control to find optimal controls taking account of routing reactions. Recently, Liu and Smith (2015), and Smith (2015) investigated the combined traffic assignment model taking into account the route choice and traffic signal control at the same time. Specifically, Liu and Smith (2015) introduced a new combined traffic assignment process known as the restricted proportional adjustment process (RPAP). The signal control model may be regarded as a model of a system periodically updated either by an operator or by an automatic system. Smith (2015) showed how route choice and signal control work simultaneously. Initially, green times give rise to travel times that influence travelers choice of route. After intersection and road delay are modified according to the assigned traffic flowflow, the control policy is recalculated to update signal green times. Thereafter, a revised control policy is adapted again. Finally, the process ends when the control policy and traveler route choice become mutually consistent.

In this study, the microscopic and macroscopic models are used simultaneously to test stability to evaluate macroscopic traffic assignment processes. Many studies proved that the dynamical model of route choice is globally stable. In the field of dynamical model of route choice, Smith (1984) applied the Lyapunov theorem, the stability tests at the fixed points of a nonlinear system, to prove the global stability of combined traffic assignment for the first time. He showed that in the dynamical model of route choice, traffic flowflows converge to the set of Wardrop equilibria with time, and proved that the global stability of traffic assignment is stable if path costs are differentiable and monotone. Subsequently, Lee (1995), Ghali (1995), Meneguzzer (1996), He, Guo, and Liu (2010), Liu and Smith (2015), Smith (2015), Smith and Watling (2016), and other numerous researchers used, developed, and modified the Lyapunov theorem in the stability test of the dynamical model of route choice. Zhang, Ge, and Hang (2000), Boyer, Blandin, and Wynter (2015), and Jia and Ngoduy (2016) used the Lyapunov theorem to prove the stability of microscopic models (ACC, CACC, V2X), and routing strategies. 
On the other hand, much research on the traffic flow model introduced a string stability test for traffic flows. Swaroop (1997) introduced the string stability of interconnected systems and established sufficient conditions to guarantee string stability. Treiber and Kesting (2013) classified the degree of instability with four stability types: stable, string metastable, linearly string unstable, and locally unstable. Treiber and Kesting also proved the car-following models condition for each string stability type. Milanes et al. (2014) and Naus et al. (2009) related these string stability conditions to the ACC and CACC CV environments.

In the field of stability, there exist string stability at the microscopic level and Lyapunov stability at the macroscopic level. The presence of only one of these two cannot guarantee user equilibrium in network: string stability applies to traffic flows within a link whilst Lyapunov stability applies to route flows in the network. Unless both string stability and network stability obtain, the stability of the aggregated model is not guaranteed. In the present paper, we therefore focus on both string stability and network stability concurrently.

The objective of this study is to examine the stability analysis of CV environment using a dynamical model of route choice based on traffic flow modelling. However, it is hard for the conventional traffic assignment model to evaluate the $\mathrm{CV}$ environment because of its microscopic properties of CV environment. Therefore, this study attempts to build an aggregation model of car-following model and the dynamical process of day-to-day rerouting. With this aggregation model, stability is mathematically formulated and analyzed by graphing the convergence of path costs.

\section{Nomenclature}

$v_{i} \quad$ Speed of vehicle $i$

$v_{\text {safe }}$ Safe speed

$v_{e} \quad$ Equilibrium speed

$\Delta t \quad$ Time step

$s_{i} \quad$ Position of vehicle $i$

$a_{i} \quad$ Acceleration of vehicle $i$

$C_{d} \quad$ Desired gap between a vehicle and the preceding one

$\tau \quad$ Desired time gap between a vehicle and the preceding one

$C_{i} \quad$ Real gap between vehicle $i$ and the preceding one

$\dot{C}_{i} \quad$ Relative speed between vehicle $i$ and the preceding one

$S S D$ Stopping sight distance

$X(t)$ Number of vehicles at time step $t$

$C_{s}(X)$ Link travel cost of link $l$

$\Delta_{r s} \quad$ Route swap vector from $r$ to $s$

$v_{l}(t)$ Average speed of vehicles in link $l$

$X_{l}^{\text {cap }}$ Link capacity at link $l$

$L_{l} \quad$ Length of link $l$

$\Phi(X)$ Differentiate function of $X$

$V(X)$ Lyapunov function of $X$

$\mathcal{N}$ A set of nodes

$\mathcal{L} \quad$ A set of links

$\mathcal{G}$ A directed graph, $\mathcal{G}=(\mathcal{N}, \mathcal{L})$

$C$ The set of lrigin-destination(OD) pairs $(p, q), C \subset \mathcal{N} \times \mathcal{N}$

$\mathcal{R}$ The set of routes $r \in \mathcal{R}_{p q},(p, q) \in C$

$\pi \quad$ OD pair least travel costs $\pi \in \mathcal{R}^{|C|}$

$d \quad$ Vector-valued demand function of the least travel costs $d=g(\pi)$

$\Gamma \quad$ Route-OD pair incidence matrix $\Gamma \in \mathcal{R}^{|\mathcal{R}| \times|\mathcal{C}|}$

$\gamma_{i} \quad$ positive control parameters of $i=1,2$

$\delta \quad$ Time-varying communication delay

$s_{i} \quad$ The sum of the real position and ideal position of vehicle $i, x_{i}=s_{i}+i \times v_{i} \times \tau$ 
Based on the traffic flow model under CV environment, we evaluate the cost of each link to choose the best route for each vehicle. The swapping process used simplified version of the restricted proportional adjustment process (SPAP) to apply to $\mathrm{CV}$ environment.

The main contributions of this study are threefold.

- This study considers how such component models can be aggregated. Then, we evaluate the stability of traffic assignment presented in this study. The dynamical models of route choice considering the intersection delay under CV environment will contribute toward effective traffic demand management and predict the link traffic flowflows properly under CV environment.

- This study suggests appropriate examples of the combined traffic assignment model considering macroscopic and microscopic effects together. The $\mathrm{CV}$ environment case can also be used by the aggregation model.

- The dynamical model of route choice using microscopic model suggested by this study, allows evaluation of new technologies that are difficult to achieve with either microscopic or macroscopic models alone.

This paper is organized as follows. Section 2 overviews the traffic flow model, dynamical model of route choice, and their application to the $\mathrm{CV}$ environment. Section 3 explains the overall stability test, Lyapunov theorem, and string stability, and their application to the CV environment. Section 4 presents numerical experiments and results. Finally, Section 5 concludes this study and discusses the outlook.

\section{The dynamical model of route choice}

\subsection{Dynamical model of route choice}

Ran and Boyce (1996) described a dynamical model of route choice. Drivers who travel from the origin to the destination require the combination of modes, departure times, and routings that serves them best. Based on this description of drivers needs, they identified four possible objectives:

- Each driver seeks to minimize his or her actual travel time, given a specified departure time.

- Each driver seeks the current best route at each intersection for current traffic conditions, at that time.

- Each driver agrees to accept a route at a specified departure time that minimizes the travel time of all vehicles traveling during a longer time period.

- Each driver agrees to accept a route at a specified departure time that minimizes the travel time of all vehicles traveling during a longer time period.

Each of these objectives is conceptually based on the purposes of this study. The CV environment notices traffic congestion and provides route control for efficiency of traffic flows in real time, though we do not consider mode choice in this study. Therefore, in this study, excluding the fourth objective, the description of the drivers needs can be modified as follows:

- CV driver seeks to minimize his or her actual travel time, interacting with other vehicles and infrastructures.

- CV driver seeks the current best route at each intersection for real-time traffic conditions, at that time.

- CV driver agrees to accept a route in real time that minimizes the travel time of all links traveling during a longer time period.

Until now, the traffic flow model is hard to describe the route choice behavior and dynamical model of route choice is hard to describe $\mathrm{CV}$ drivers behavior. Therefore, an expression of dynamical model of route choice using the traffic flow model is needed to describe the CV environment. Describing the CV environment using the traffic flow model, route choice is the best way to assign traffic flow. 


\subsubsection{Dynamical model of car-following model}

Since transportation engineering has been established, there are many microscopic car-following models. Above all, car-following models for the purpose of maintaining a safe gap between the leading vehicle and following vehicle are commonly used to simulate CV environment. Gipps model and the Intelligent Driver Model (IDM) are examples of these car-following models. This study uses a simplified version of the Gipps model (Gipps, 1981). Treiber and Kesting (2013) explain that the simplified Gipps model is just simplified, not essentially changed.

Yu and Shi (2015), physics researchers, also studied an improved car-following model considering changes in the relative gap between vehicles. An improved car-following model is composed of relative speed affected by relative gap. Numerous car-following models are classified depending on the kind of variable under consideration, including speed, relative speed, and gap.

Recent research about microscopic models in the field of traffic engineering concentrate on acceleration. Jia and Ngoduy (2016) design a suitable vehicle control and propose an improved consensus-based control algorithm for the ACC considering V2X communications in a car-following model. This car-following model calculates acceleration by using the sum of relative gap and velocity gap.

\subsubsection{Dynamical model of route choice}

The dynamical model of route choice is different from the conventional traffic assignment problem. Here, we adopt the variational inequality formulation: A feasible assignment $X *$ is an equilibrium if, for all feasible assignments $X$

$$
-C\left(X^{*}\right) \cdot\left(X-X^{*}\right) \leq 0
$$

where $X^{*}$ is the equilibrium point of traffic volume

Marcotte and Patriksson (2007) presented Wardrops equilibrium principle using variational inequalites in a more geometric shorthand route-based notation in the vector space.

Theorem 1. (Marcotte and Patriksson, 2007) The variational inequality problem is expressed by

$$
\begin{aligned}
& 0^{|\mathcal{R}|} \leq X \perp(C(X)-\Gamma \pi) \geq 0^{|\mathcal{R}|} \\
& \Gamma^{T} X=d \\
& d=g(\pi)
\end{aligned}
$$

\section{Proof. See the proof in Appendix A.}

To consider traffic signals or route choices for each time step, we adopt the Proportional Adjustment Process (PAP) as a suitable method. The following expressions are equivalent to the Wardrop equilibrium condition, not conceptually changed, (Liu and Smith, 2015).

- Travelers will stay on the same route if there is no cheaper route.

- If there is a cheaper route, then some travelers will periodically switch to one.

The rate of the change of flow is specified by a function that describes the swapping among alternative paths and is followed by the following process.

- The flow $X_{r}(t)$ on the more expensive route $r$ on day $t$.

- The difference $C_{r}(X(t))-C_{s}(X(t))$ in route costs on day $t$.

$X(t)=\left[X_{r}(t), X_{s}(t)\right]$ is the route-traffic flow vector on day $t$ and $C(X(t))=\left[C_{r}(X(t)), C_{s}(X(t))\right]$ is the route-cost vector on day $t$. The basic assumption of the swapping process is that the traffic flow swapping from one route to the other route will be proportional to the product of the two factors. However, this study attempts to combine microscopic models with macroscopic methods to eliminate swapping specific traffic volumes. We just need one vehicle to choose another route for each time step $\Delta t$. Therefore, the product of constant $k, X^{0}$, and swapping flow vector $\Delta_{r s}(t)$ is 
omitted for abbreviation. We only need swapping vectors $\Delta_{r s}(t)$ and $C(X(t))$ in the vehicle swapping process. We call this process the Simplified Proportional Adjustment Process (SPAP), which is expressed by the following formula:

$$
\begin{aligned}
& X(t+\delta t)=X(t)+\Delta_{r s}(t) \quad \text { for all } t=1,2,3, \cdots \\
& X(0)=X^{0} \\
& X(t)=X^{0}+\sum_{k=1}^{t} \Delta_{r s}((k-1) \times \Delta t) \\
& \text { where } \Delta_{r s}(t)=\left\{\begin{array}{l}
+1 \text { if } C_{s}(X(t-1)) \leq C_{r}(X(t-1)) \\
-1 \text { if } C_{s}(X(t-1)) \geq C_{r}(X(t-1))
\end{array}\right.
\end{aligned}
$$

For example, if at time $(t-1)$ the cost on route 2 is greater that that on route 3 , then the swap vector from rotue 2 to route 3 at time $\mathrm{t}$ will be $\Delta_{23}(t)=[-1,1]=(-1,1)^{T}$.

\subsection{Dynamical model of route choice under CV environment}

To express the CV environment, many components are considered. In this study, we concentrate on two components: ACC and radio communication between the vehicle and infrastructure.

\subsubsection{Dynamical model of car-following model under CV environment}

Adaptive Cruise Control (ACC) is introduced to prevent rear-end collision . Milanes et al. (2014) assumed that for the ACC system, headways range from 1.1 seconds to 2.2 seconds. By using lidar or radar to monitor the preceding vehicle, ACC makes each vehicle adjust its speed towards that of any preceding vehicle at a suitable spacing. We express the acceleration as $a_{i}=\ddot{x}_{i}$ i, the desired headway between the preceding and following vehicle as $C_{d}$, real headway as $C_{i}$, real relative speed at $i$ th and $i-1$ th vehicle as $v_{i}^{r}=\dot{C}_{i}$, Stopping Sight Distance as $S S D$, and parameters as $K$. Then the acceleration of vehicle $i$ can be expressed as follows.

$$
\begin{aligned}
& a_{i}=\ddot{x}_{i}=-K_{1}\left(C_{d}-C\right)-K_{2} v_{i}^{r}, \quad i \geq 2 \\
& \text { where } C_{d}=v_{i-1} \times \tau+S S D
\end{aligned}
$$

Fig. 1 depicts the ACC system under the CV environment. A preceding vehicle and a V2X vehicle, a nearby $\mathrm{V} 2 \mathrm{X}$ vehicle are considered as variables to explain the behavior policy of the vehicle. Communication delay and information from RSU is also considered. By applying a preceding gap error controller, we keep the headway control between a vehicle and the preceding one.

\subsubsection{Dynamical model of route choice under CV environment}

Dynamical modelling is appropriate for route choice. V2I communication can relay the downstream traffic conditions and important information related to road safety. Relaying traffic information related to traffic signals and road condition can help drivers judge the driving behavior and road condition better. The CV and V2I algorithms are only effective within their specific ranges. Widely used radio communication technology under the CV environment is shifting from DSRC (Dedicated short-range communications) to WAVE (Wireless Access in Vehicular Environment) and IEEE $802.11 \mathrm{p}$ because of the range and benefits of radio communication technology. The maximum effective range of WAVE is $1,000 \mathrm{~m}$ (average $300500 \mathrm{~m}$ ).

In summary, vehicles under the $\mathrm{CV}$ environment feature an aggregate of many new technologies. However, in this study, we consider two specific new technologies, V2I and ACC. The block diagram in Fig. 1 shows the flow of information and feedback process. To calculate the kinematic information of each vehicle such as position and acceleration, information from the preceding vehicle, leading vehicle, upstream CV vehicle, and RSUs are needed. An aggregation between microscopic and macroscopic model algorithms is suggested to evaluate stability and how the link flows converge to equilibrium. 


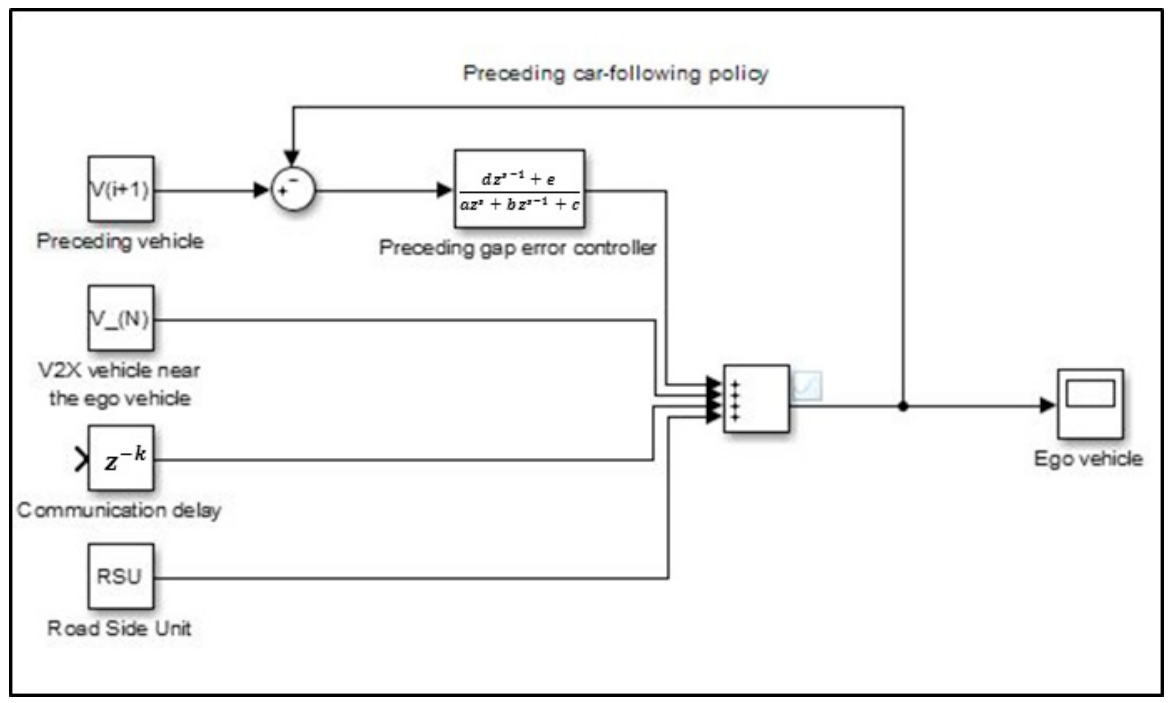

Fig. 1. Block diagram of the CV environment

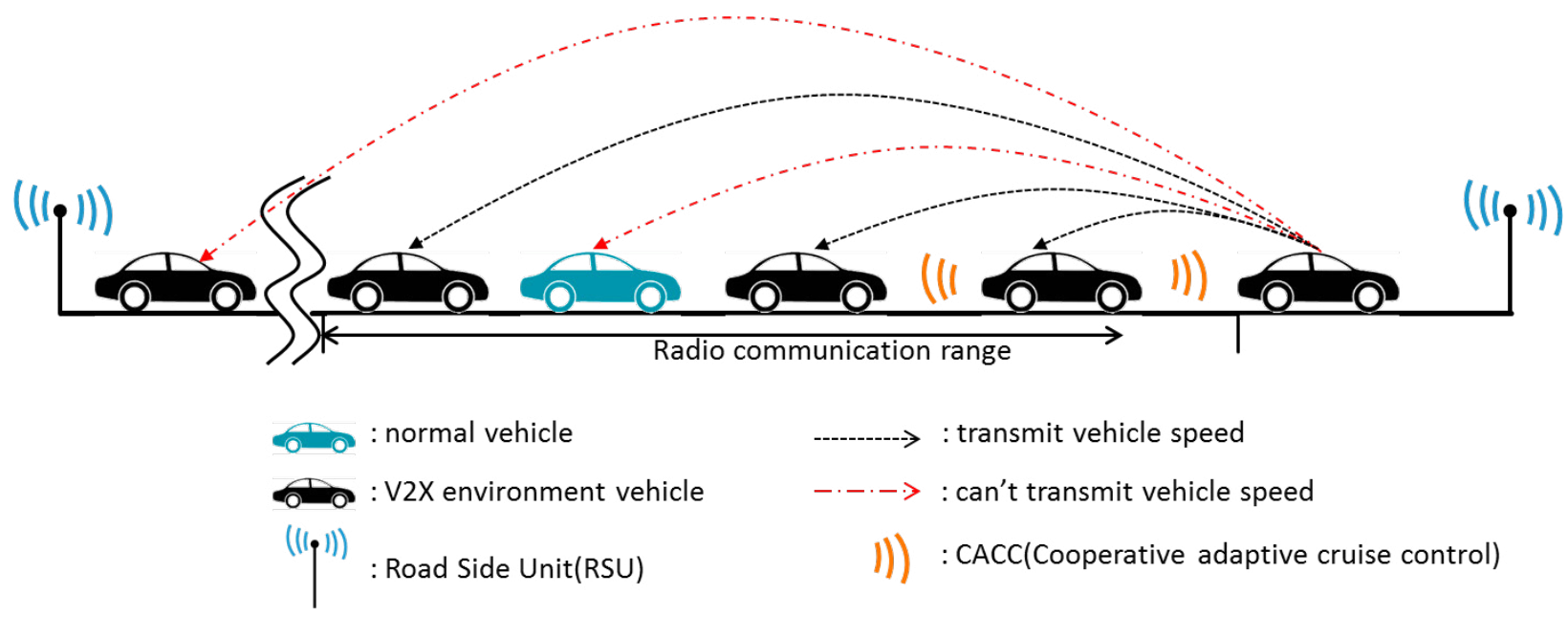

Fig. 2. Description of CV environment

\section{Stability analysis on a dynamical model of route choice}

\subsection{Stability analysis on the car-following model}

\subsubsection{String stability test}

To test stability, the string stability test is used. Milanes, et al. (2014) and Swaroop (1994) defined string stability as reduction of perturbations downstream traffic flows, preventing that dynamic perturbation due to preceding vehicle speed reduction causes in the downstream vehicles. To keep the desired headway, previous equations are arranged by $C_{i}$, we obtain:

$$
\begin{aligned}
& \ddot{x_{i-1}}=-K_{1}\left(v_{i-1} \times \tau+S S D-\left(x_{i-2}-x_{i-1}\right)\right)-K_{2}\left(v_{i-2}-v_{i-1}\right) \\
& \ddot{x}_{i}=-K_{1}\left(v_{i} \times \tau+S S D-\left(x_{i-1}-x_{i}\right)\right)-K_{2}\left(v_{i-1}-v_{i}\right) \\
& x_{i-1}-\ddot{x}_{i}=-K_{1}\left(\tau \times\left(v_{i-1}-v_{i}\right)-\left(C_{i-1}-C_{i}\right)\right)-K_{2}\left(v_{i-1}^{r}-v_{i}^{r}\right) \\
& \ddot{C}_{i}+\left(K_{2}-K_{1} \times \tau\right) \times \ddot{C}_{i}+K_{1} C_{i}-K_{2} C_{i-1}-K_{1} C_{i-1}=0 \quad i \geq 2
\end{aligned}
$$


Table 1. Proposed aggregation model algorithm for evaluating stability.

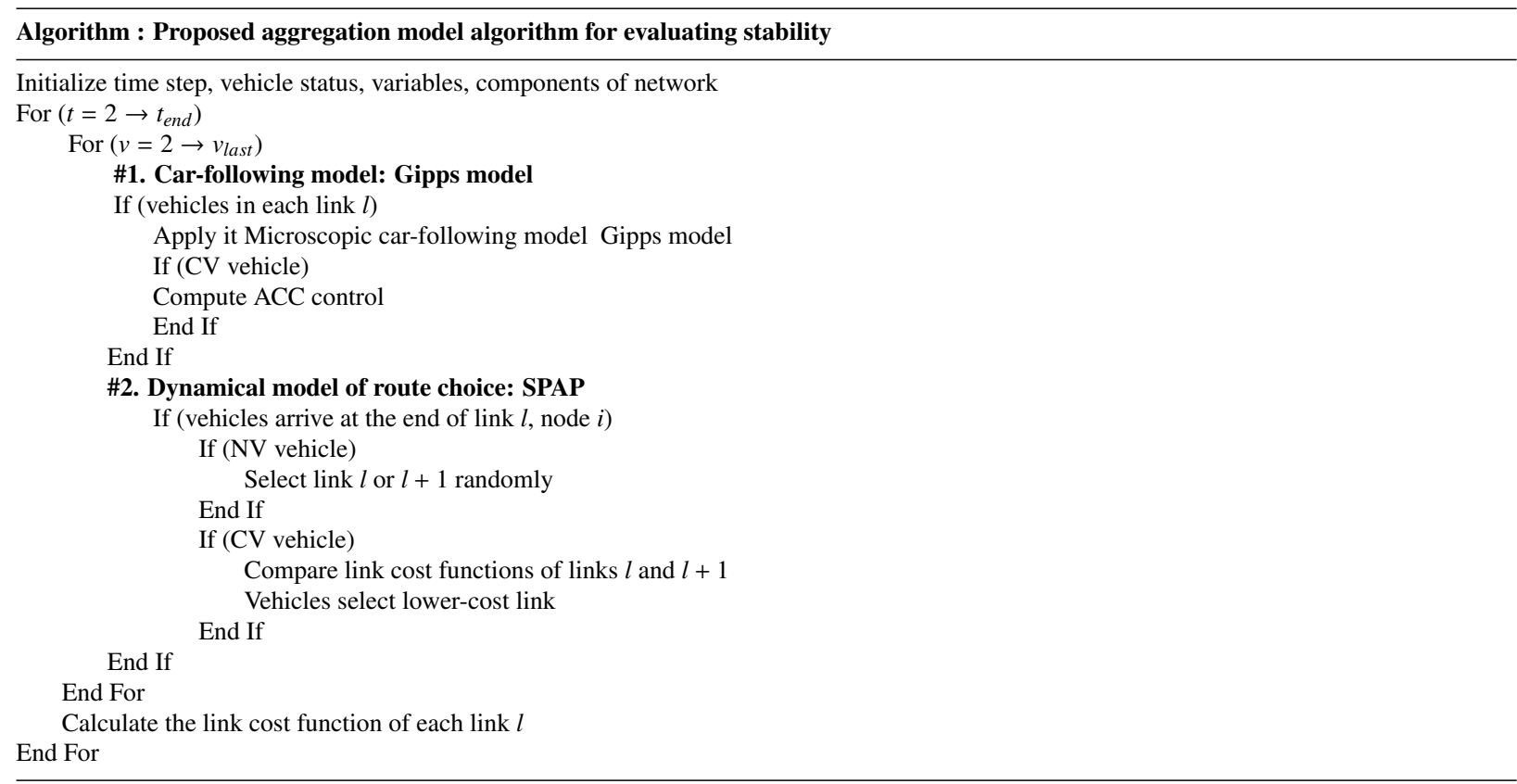

Solving these ordinary differential equations, we obtain the following formula:

$$
|S S(s)|=\left|\frac{c_{i}}{c_{i-1}}\right|=\frac{-K_{2} s+K_{1}}{s^{2}+\left(K_{1} \tau-K_{2}\right) s+K_{1}} \quad i \geq 2
$$

Theorem 2. Traffic flow is strng stable if $|S S(s)| \leq 1$

As a result, ACC can help relieve traffic perturbation and improve traffic flow. Vehicles under CV environment can reduce their headways and change the speed smoothly so that traffic flows can be improved.

The stability of the Gipps model is guaranteed by the string stability test, so that perturbations decay over time.

Theorem 3. Treiber and Kesting (2013) explain that the Gipps model satisfies string stability if the following criterion is satisfied.

$$
\frac{1}{\Delta t} \leq \frac{1}{2 \Delta t}\left(1+\frac{v_{e}}{v_{e}+b \Delta t}\right)
$$

Proof. The proof of this criterion is presented in Appendix $B$.

\subsubsection{Lyapunov stability test}

Besides string stability test, the Lyapunov stability test is commonly used to prove the traffic flow model is stable. Jia and Ngoduy (2016) applied V2I algorithm to a microscopic model combined with the CV algorithm, and proved the models stability using a non-linear stability test, the Lyapunov theorem.

Theorem 4. (Jia and Ngoduy, 2016) If there exists a spanning tree in the topology graph $\mathcal{G}=(\mathcal{N}, \mathcal{L})$, under the $C V$ algorithms, and the control parameters $\beta_{1}, \beta_{2}$ satisfy

$$
\left[\begin{array}{cc}
\beta_{1}^{2}\left(L+L^{T}\right)-\beta_{2} I & \beta_{1} \beta_{2}\left(L^{T}+I\right)-\left(\beta_{1}+\beta_{2}\right) I \\
\beta_{1} \beta_{2}(L+I)-\left(\beta_{1}+\beta_{2}\right) I & \left(2 \beta_{2}^{2}-2 \beta_{1}-\beta_{2}\right) I
\end{array}\right]
$$

Then the state error between the following vehicle and the downstream reference is uniformly ultimately bounded by:

$$
\lim _{t \rightarrow \infty}\left\|x_{k}\right\| \leq C_{0}
$$

Proof. See the proof in Jia and Ngoduy (2016). 


\subsection{Stability analysis on a dynamical model of route choice}

\subsubsection{Lyapunov stability test}

A traffic assignment model is globally stable for a certain problem if any initial flows in the feasible region lead to an equilibrium by a dynamical adjustment process of this model. Using Lyapunovs theorem (see, for example, Zangwill 1969), Smith (1984) was able to show that user equilibrium is stable if path costs are differentiable and monotone.

The stability of SPAP is equal to the stability of RPAP suggested by Liu and Smith (2015), who used the Lyapunov stability test originated from Smith (1984).

$$
\dot{X}(t)=\Phi(X(t)) \quad \text { for } t>0
$$

The dynamical system is stable if and only if, for any $X_{0} \in D$, the solution of preceding equations with $X(0)=X_{0}$ converges as $t \rightarrow \infty$. Let $\Phi$ be continuously differentiable, then the dynamical system is stable if there is a continuously differentiable scalar function $V(\cdot)$ defined in $D$, such that

- $V(X) \geq 0$ for all $X \in D$

- $V(X)=0$ if and only if $X$ is an equilibrium, and

- $\frac{d V(x)}{d X} \Phi(X)<0$ if $X$ is not an equilibrium

where $D=\left\{X \mid X \in R^{n}\right.$ with $A X=q$ and $\left.X \geq 0\right\}$ : demand-feasible route flows, $S=\left\{X \mid X \in R^{n}\right.$ with $\left.X \geq 0\right\}$

Definiiton $x^{*}$ is said to be a locally exponentially stable equilibrium point if there exists $\mathrm{h}$ small enough and $m, \gamma>0$, such that

$$
\left|x(t)-x^{*}\right| \leq m\left|x_{0}-x^{*}\right| e^{-\gamma t}
$$

For all $x \in B_{h}\left(x^{*}\right)$ and $t>0$.

The following theorem provides sufficient conditions for an equilibrium $x^{*}$ to be locally exponentially stable.

Theorem 5. (Sastry, 1999): An equilibrium point $x^{*}$ is locally exponentially stable if there exists a differentiable function $V(X),\left(\alpha_{1}, \alpha_{2}, \alpha_{3}, \alpha_{4}\right) \in \mathbb{R}_{+}^{* 4}$ and $h \in \mathbb{R}_{+}^{*}$ such that $\forall x \in B_{h}\left(x^{*}\right)$

- $\alpha_{1}\left|x-x^{*}\right|^{2} \leq V(X) \leq \alpha_{2}\left|x-x^{*}\right|^{2}$ (V is locally quadratic.)

- $\left|\nabla_{X} V(X)\right| \leq \alpha_{4}\left|x-x^{*}\right|\left(V\right.$ evlovles slowly around $x^{*}$.)

- $\left(\nabla_{X} V(X)\right)^{T} f(x) \leq-\alpha_{3}\left|x-x^{*}\right|^{2}$ (The system evolves toward argmin $V(X)$.)

\section{Numerical experiments}

As a preliminary to the numerical experiments presented here, three important assumptions are made in the microscopic model.

- There is not data loss within the radio communication systems.

- Under CV environment, only ACC and V2I technologies are considered. In this study, we focused on the stability analysis of the network, so we did not consider other technologies such as CACC and lane changing behavior.

- String stability is used for a limited length under CV environment. Treiber and Kesting (2013) define string stability as Traffic flow is string stable if local perturbations decay everywhere even in arbitrarily long vehicle platoons. Otherwise, it is string unstable. This means that long vehicle platoons, as in congested conditions, require string stability.

Two assumptions are made in the macroscopic model of route choice. 
- Swapping flow vector used by SPAP process only considers one vehicle at each time step because of its limited scale.

- The macroscopic model is considered as a small time step. In fact, RPAP is a day-to-day rerouting process. This means that the macroscopic time step is set to a typical value of one day. However, in the calculation process, the scale is not relevant; therefore, we assume that the macroscopic time step is set to one second, same as that of the microscopic time step.

\subsection{Numerical experiments - A two-route network}

\subsubsection{Car following model settings - Gipps' model}

The simplified Gipps model calculates a vehicles speed with previous vehicles speed $v$, acceleration $a$, initial speed $v_{0}$, and safe speed $v_{0}, v_{s a f e}\left(s, v_{l}\right)$. In Gipps model, each vehicle maintains assumes a safety gap between behind its leading and following vehicle by using $v_{s a f e}\left(s, v_{l}\right)$, which is originated calculated from the stopping distance. After calculating these variables, the model chooses the minimum of them and adds the error term to reflect the difference of safe speed distance of each driver. These equations (Treiber and Kesting, 2013) are as follows:

$$
\begin{aligned}
& v_{i}(t+\Delta t)=\min v_{i}+a \Delta t, v_{0}, v_{i_{\text {safe }}}\left(s, v_{l}\right)+\varepsilon \\
& \text { where } v_{\text {safe }}\left(s_{i-1}, v_{i-1}\right)=-b \Delta t+\sqrt{b^{2} \Delta t^{2}+v_{i-1}^{2}+2 b\left(s_{i-1}-s_{0}\right)} \\
& a_{\text {mic }}^{\text {Gipps }}\left(s_{i-1}, v_{i}, v_{i-1}\right)=\frac{\left.v_{\text {safe }}\left(s_{(} i-1\right), v_{i-1}\right)-v_{i}}{\Delta t}=\frac{\sqrt{b^{2} \Delta t^{2}+v_{i-1}^{2}+2 b\left(s_{i-1}-s_{0}\right)}-b \Delta t-v_{i}}{\Delta t} \quad i \geq 2
\end{aligned}
$$

To model vehicle behavior, Gipps car-following model is used. Lane-changing behavior is not considered in this study so we simplify that every link has one lane. We assume that there are few signals and interchanges, so we apply the suggested model to highway value.

The dynamical model of route choice is also calculated for each time step $\Delta t_{\text {macro }}$. This study aggregates Gipps car-following model and a dynamical model of route choice for each same time step $\Delta t_{\text {micro }}=\Delta t_{\text {macro }}$. We assume that SPAP is only applied to connected vehicles, and the other normal vehicles choose the route randomlyindependently, so that . Thus, the market penetration rate (MPR) of connected vehicles is considered important.

Table 2. Parameters of the simplified Gipps' model (Treiber \& Kesitng, 2013)

\begin{tabular}{lll}
\hline Parameter & Typical value highway & Typical value city traffic \\
\hline Desired Speed & $120 \mathrm{~km} / \mathrm{h}$ & $54 \mathrm{~km} / \mathrm{h}$ \\
Adaption/reaction time & $1.1 \mathrm{sec}$ & $1.1 \mathrm{sec}$ \\
Acceleration & $1.5 \mathrm{~m} / \mathrm{s}^{2}$ & $1.5 \mathrm{~m} / \mathrm{s}^{2}$ \\
Deceleration & $1.0 \mathrm{~m} / \mathrm{s}^{2}$ & $1.0 \mathrm{~m} / \mathrm{s}^{2}$ \\
Minimum distance & $3 \mathrm{~m}$ & $2 \mathrm{~m}$ \\
\hline
\end{tabular}

\subsubsection{Dynamical model of route choice settings Simplified Proportional Adjustment Process}

Gipps model is used adopted here as the car following model too update the state (position and speed) of. Carfollowing models in microscopic traffic flow models depict each vehicles status for at each time step $\Delta t_{\text {micro }}$.

In this study, traffic dynamic system is applied to a two-route network. Fig. 4 depicts its two-route network. Vehicles are generated at node 0 and travel to node 3 . At node 1 , each vehicle has to choose between link 2 and link 3. Then, we evaluate the links at the end of each time step by using flow delay function, which originates from the Bureau of Public Road (BPR), to calculate the generalized travel cost. This BPR function is suitable for evaluating link cost from traffic flow. This study adopts as the variable of vehicle speed. According to Gipps model, $v_{l}(t)$ will approach the free-flow speed in the case of low traffic flow, and so will avoid any abrupt speed reduction. We adopt the BPR function to relate link travel times to traffic:

$$
C_{l}\left(v_{l}, \mathbf{X}\right)=f_{l}\left(v_{l}(t), X(t)\right)=\frac{L_{l}}{v_{l}(t)}\left[1+\alpha\left(\frac{X(t)}{X_{l}^{c a p}}\right)^{\beta}\right]
$$




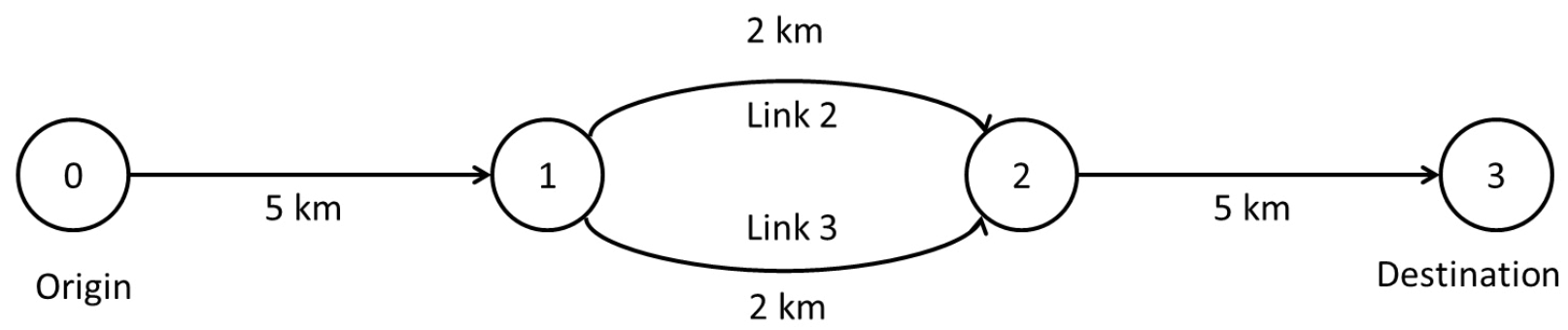

Fig. 3. A two-route network

After we calculate each routes cost, we calculate the route cost difference between the two and choose the cheaper one. The difference of this cost function will converge to zero by using the SPAP. We will investigate the stability of this system by testing the process.

To evaluate V2I, variables that affect V2I are considered, of which market penetration rate (MPR) of CV vehicles is one of the main variables of interest. If a vehicle is not connected (we call it a normal vehicle, NV), the probability that the route choice of $\mathrm{NV}$ vehicles (P(TRUE)) is equal to the route choice of $\mathrm{CV}$ vehicles is defined as follows:

$$
\begin{aligned}
P(T R U E) & =P(T R U E \cap C V) \\
& =P(C V) P(T R U E \mid C V)+P(N V) P(T R U E \mid N V) \\
& =M P R(1-\varepsilon)+(1-M P R) \alpha \\
& =M P R(1-\varepsilon-\alpha)+\alpha
\end{aligned}
$$

where transmission failure rate is $\varepsilon$, then $P(T R U E \mid C V)=1-\varepsilon$, and $P(T R U E \mid N V)=\alpha$

For the sake of simple illustration, we set $\alpha=\frac{1}{2}$ and $\varepsilon=0$, so that

$$
\begin{aligned}
& P\left(T R U E^{\prime}\right)=\frac{1+M P R}{2} \\
& P\left(F A L S E^{\prime}\right)=\frac{1-M P R}{2}
\end{aligned}
$$

If this probability is applied to the flow swapping vector used by SPAP, the equation obtained is as follows:

$$
\begin{aligned}
& \Delta_{r s}^{M P R}(t)=\Delta_{r s}(t) \cdot P(T R U E)=\Delta_{r s}(t) \cdot \frac{1+M P R}{2} \\
& \Delta_{r s}^{M P R}(t)= \begin{cases}\frac{1+M P R}{2} & \text { if } C_{s}(X(t-1)) \geq C_{r}(X(t-1)) \\
-\frac{1+M P R}{2} & \text { if } C_{s}(X(t-1))>C_{r}(X(t-1))\end{cases} \\
& X(t+\Delta t, M P R)=X(t, M P R)+\Delta_{r s}^{M P R}(t), \quad \text { for all } t=1,2,3, \cdots \\
& X(t, M P R)=X^{0}+\sum_{k=1}^{t} \Delta_{r s}((k-1) \times \Delta t) \cdot \frac{1+M P R}{2}
\end{aligned}
$$

To prove the stability of SPAP under V2I components of CV environment, we use the Lyapunov theorem. In outline, the Lyapunov process follows Liu and Smith (2015). Let $r \sim s$, which means that routes $r$ and $s$ join the same origin-destination pairOD: $r, s \in P_{O D}$. We define the SPAP-modified Lyapunov objective function $V$ by restricting the route pairs $(r, s)$ as:

$$
V(X(t))=\sum_{\{(r, s) ; r \sim s\}} X_{r}(t)\left(C_{r}(X(t))-C_{s}(X(t))\right)_{+}^{2} \quad \text { for all } X \in D
$$

where $D$ is the demand - feasible set : $D=\left\{X(t) \mid \sum_{\left\{r \in P_{O D}\right\}} X_{r}(t)=\rho_{O D} \quad \forall O D, \quad X_{s}(t) \geq 0, \quad \forall s\right\}$ 
Then, $V$ is a measure of departure from equilibrium. It is easy to see that for $X \in D$.

$V\left(X(t)=0 \quad\right.$ if and only if $\left\{\right.$ for all $r, s$ such that $\left.r \sim s, C_{r}(X(t))-C_{s}(X(t))>0 \Rightarrow X_{r}=0\right\}$

if and only if $\left\{\right.$ for all $r, s$ such that $\left.r \sim s, X_{r}\left[C_{r}(X(t))-C_{s}(X(t))\right]_{+}^{2}=0\right\}$

if and only if $X$ is a Wardrop equilibrium(Wardrop, 1952) \}

The set $E$ of Wardrop equilibria may thus be specified as follows:

$$
E=\{X(t) \in D ; V(X(t))=0\}
$$

It is natural to consider approximate equilibria, so let (for any $\eta>0$ )

$$
E_{\eta}=\{X(t) \in D ; V(X(t)) \leq \eta\}
$$

We apply Liu and Smiths (2015) lemma to the SPAP, the suggested process in this study, because the property of variables and equations are all the same.

Lemma. (Liu and Smith, 2015) Assume that the link/route cost function $C(X)$ is monotone and differentiable in $D . V(X(t))$ is $C^{\prime}(X)$ is a continuous function of $X$. Then,

- $V(X(t)) \geq 0$ for all $X(t) \in D$ and is differentiable in $D$

- $V^{\prime}(X(t))$ is a continuous function of $X(t)$ in $D$; and

- $\frac{d V(X(t))}{d X} \Phi(X(t))<0$ if $X$ is not an equilibrium.

Proof. For more details and proof, see Liu and Smith (2015).

\subsubsection{Connection between the two models}

We calculate the time step of Gipps model $\left(\Delta t_{\text {micro }}\right)$ and that of dynamical model of route choice $\left(\Delta t_{\text {macro }}\right)$ simultaneously. This study integrates Gipps car-following model with the dynamical model of route choice for each time step $\Delta t_{\text {macro }}=\Delta t_{\text {micro }}=\Delta t_{\text {macro }}$. We assumed that SPAP is only applied to connected vehicles, and the other normal vehicles choose the route randomly with fixed parameter $\alpha$. Thus, the MPR of connected vehicles is considered important. Applying the traffic dynamic system to the two-route network example, we consider market penetration rates ranging from $10 \%$ to $90 \%$ to test Lyapunov stability.

The graphs in Fig. 4 show the costs on links 2 and 3 over time at different levels of MPR. For simplicity, graphs are shown for MPR of $10 \%, 30 \%$, and $80 \%$. The traffic flow on links 2 and 3 converge to similar values because links 2 and 3 have the same link properties. The vehicle speeds of each link are different only because of stochastic variation. When the first vehicle enters link 2 or 3, link costs fluctuate because no other vehicle is present. Thus, that is important to omit this range of link costs Link cost values for MPR 10\% fluctuate greatly and do not converge to any specific value: this case is near normal vehicle traffic flow. On the other hand, values of MPR of $30 \%$ and $80 \%$ do not fluctuate and converge to the mean of link cost function, even though some points change their link cost function when new vehicles enter the link. This means that vehicles under CV environment are effective in achieving equilibrium and even a relatively small MPR is effective in stabilizing network conditions in this simple network.

The results in Fig. 5 show the differenc in link costs between links 2 and 3. As stated above, the link difference at MPR $10 \%$ fluctuates most and that at MPR $80 \%$ approaches zero, thoughsmall perturbations exist. The link difference of MPR $80 \%$ points to reverse direction. It is inferred that the first vehicle is moving toward link 3 and not link 2.

To investigate stability in the presence of an incident, the speed of the 10th vehicle, which is assigned to link 2 , is intentionally reduced from $120 \mathrm{~km} / \mathrm{h}$ to $20 \mathrm{~km} / \mathrm{h}$ in the range of $400-440$ time steps $(200-220$ seconds). Fig. 6 shows the link cost function of links 2 and 3 at MPR of 10\%, 30\%, and 70\%. The link cost values at MPR 10\% slightly increase in the range of $400-440$ time steps. By contrast, those at MPR 30\% and $70 \%$ do not appear to have any great difference in the range of 400-440 time steps. The results in Fig. 7 show the difference in link cost between links 2 and 3. The link cost difference at MPR 10\% fluctuates most and the small link cost difference at MPR 30\% and $80 \%$ shows that a good degree of equilibrium is achieved. The difference between link costs increases slightly in the range of $400-440$ time steps at each MPR (especially MPR 80\%), and as time passes, the values of cost function approach 0 because of SPAP. 

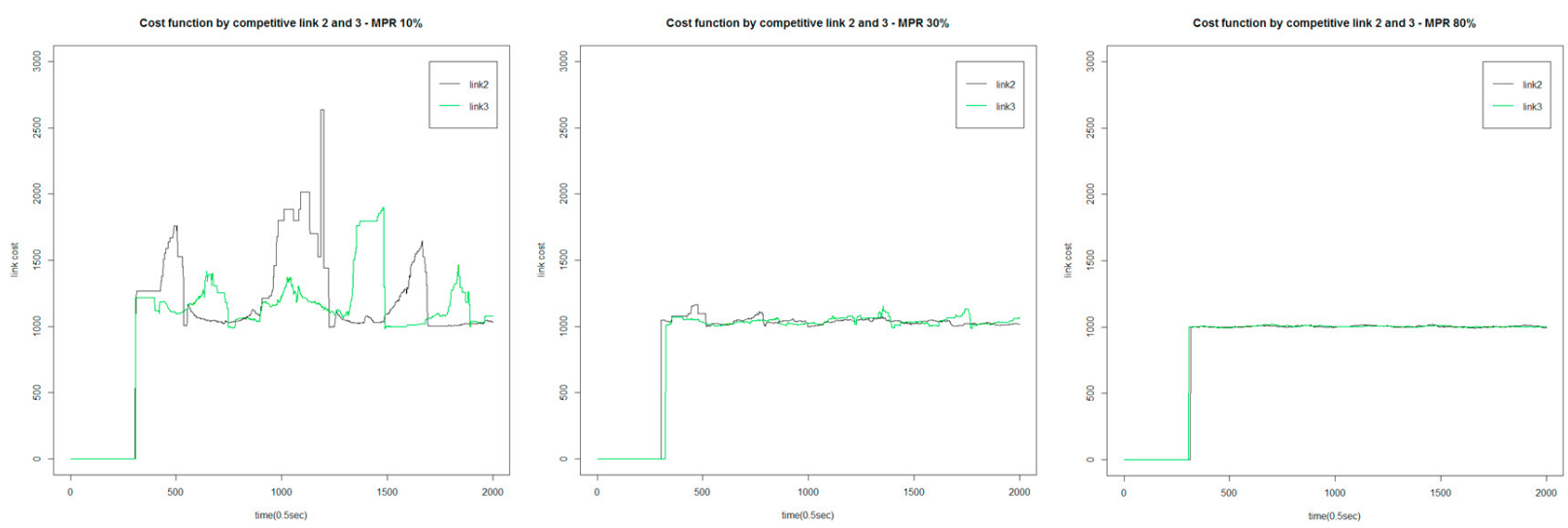

Fig. 4. Case 1 : Comparsion of link costs for market penetration rate $(10 \%, 30 \%, 80 \%)$

difference of cost by MPR ratio

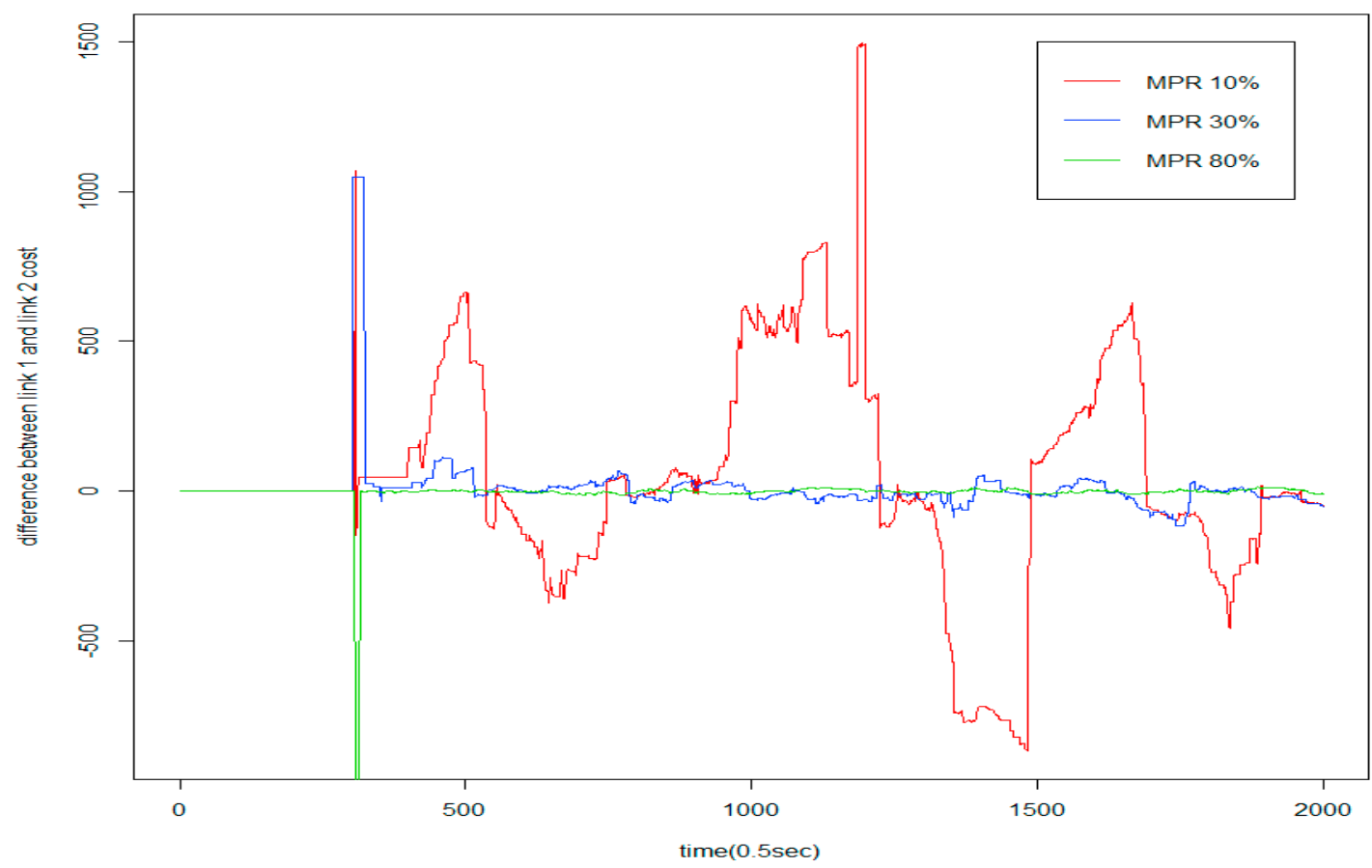

Fig. 5. Case 1 : Difference of cost by MPR (10\%, 30\%, 80\%)

\subsection{Numerical experiments - Joongbu and 2nd Joongbu expressway}

\subsubsection{Network description}

To validate the proposed algorithm, we select one direction of Joongbu and 2nd Joongbu expressway. The 2nd Joongbu expressway was constructed to increase the capacity of Joongbu expressway corridor. Joongbu and 2nd Joongbu expressway network are shown in Fig. 8. The 2nd Joongbu expressway (Link 3) is connected to other roads only at node 1 and node 8 so that traffic flow on link 3 is only affected by route 4 and route 11 . Vehicles are generated according to the route information shown in Table 2. 

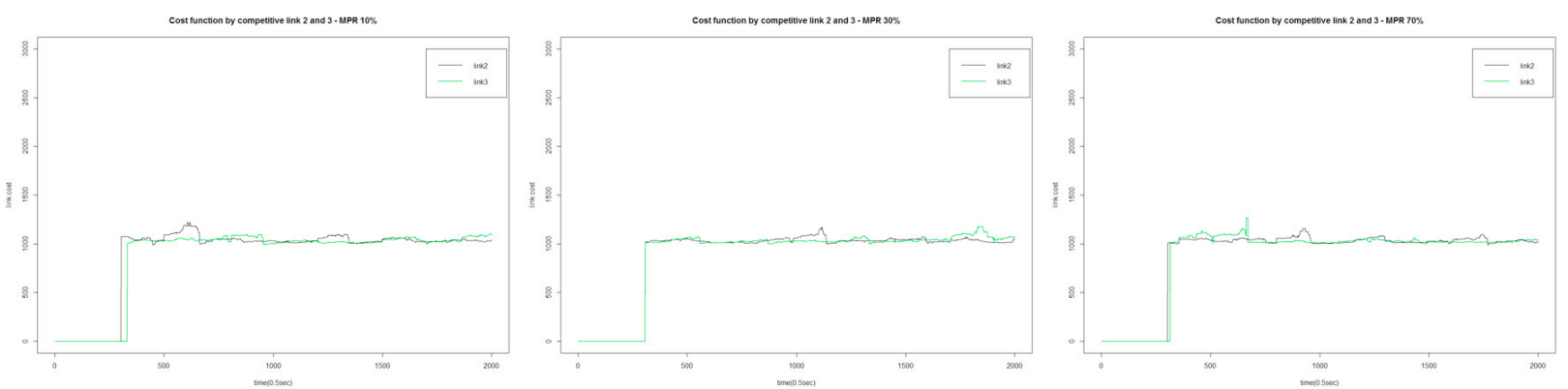

Fig. 6. Case 2 : Reduce speed to $20 \mathrm{~km} / \mathrm{h}$ at $200220 \mathrm{sec}(400440$ time steps)(10\%, 30\%, 80\%)

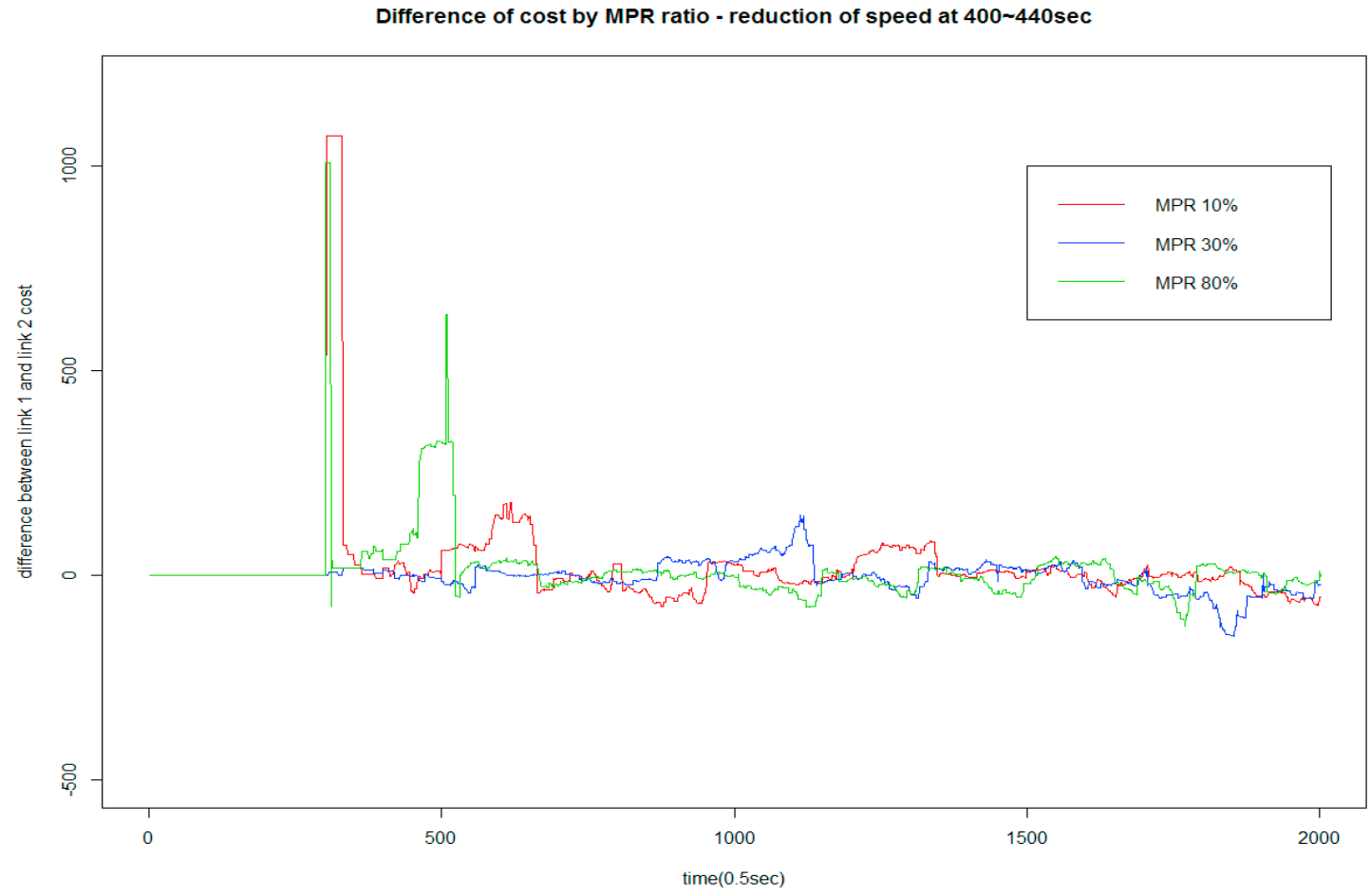

Fig. 7. Case 1 : Difference of cost by MPR (10\%, 30\%, $80 \%)$

\subsubsection{Results comparison}

The results shown in Fig. 9 show the cost values on the competing link 2 (Joongbu expressway) and link 3 (2nd Joongbu expressway). Unlike stable flow on link 3, the flow on link 2 is unstable because it is changed continuously by many routes. Due to the captive drivers of link 2, this results in a difference in costs between link 2 and link 3 .

Link cost values at MPR 10\% fluctuate throughout the range of 200400 time steps. By contrast, those at MPR $30 \%$ and $70 \%$ do not appear to have any great difference during this time. The results in Fig. 10 show the difference between costs on links 2 and 3, which are due to captive traffic on link 2. Link cost difference at MPR 10\% fluctuates most whilst those at MPR 30\% and $80 \%$ are more stable. 


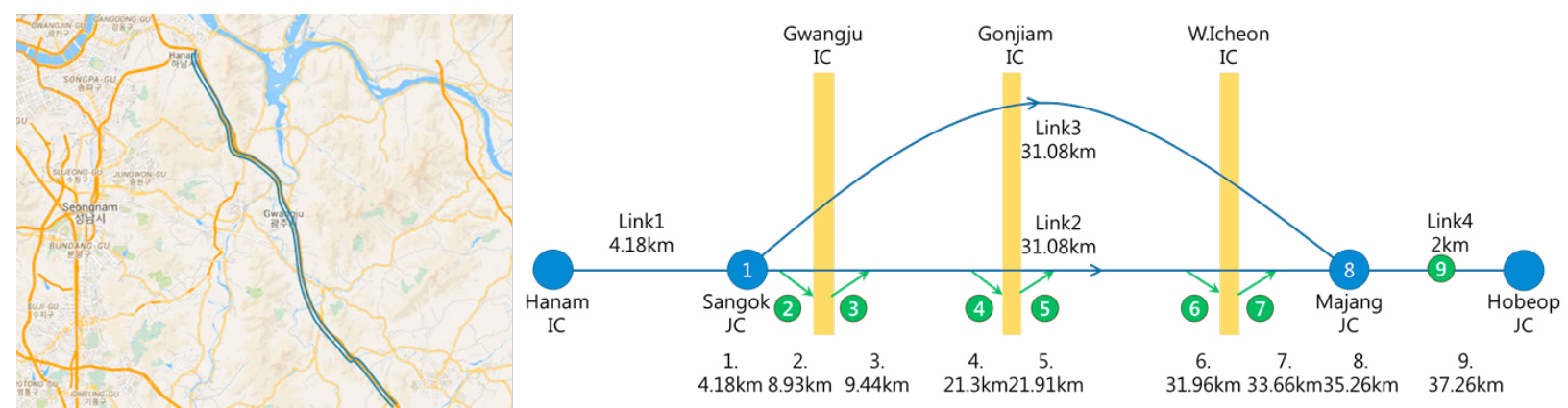

Fig. 8. Description of Joongbu and 2nd Joongbu expressway network

Table 3. Routes description of Joongbu and 2nd Joongbu expressway network

\begin{tabular}{llllll}
\hline & Origin Node & Destination Node & Lanes & Traffic flow & Mean headway(s) \\
\hline route1 & 1 & 2 & 2 & 1,244 & 2.9 \\
route2 & 1 & 4 & 2 & 39 & 92.4 \\
route3 & 1 & 7 & 2 & 213 & 17 \\
route4 & 1 & 9 & 2 & 726 & 5 \\
route5 & 3 & 4 & 2 & 3 & 1,200 \\
route6 & 3 & 7 & 2 & 2 & 1,800 \\
route7 & 3 & 9 & 2 & 2 & 50.8 \\
route8 & 5 & 7 & 2 & 31 & 9.4 \\
route9 & 5 & 9 & 2 & 387 & 144 \\
route10 & 7 & 9 & 2 & 25 & 9 \\
route11 & 1 & 9 & 204 & \\
\hline
\end{tabular}

\section{Conclusion and outlook}

This study investigates the combination of a dynamic traffic assignment model with a CV environment to study the stability of the combined system. Based on this, we evaluate each link cost to choose the appropriate route for each vehicle. The principal effects of the CV environment on the traffic assignment model are direct through information on route costs and indirect through increases in capacity. To investigatge this, we design a combination of microscopic traffic flow model and dynamic route choice model called SPAP. This model allows for optimization of travel time under the CV environment. Our results show that under the CV environment, route choice is more effective in achieving equilibrium and reaction to traffic incidents is improved. The stability of this dynamical system is helpful to convergence speed. However, due to the stochastic properties of the microscopic model, traffic flow does not converge. Therefore, we investigate the difference between link costs. This will also be useful both to represent reality and to promote the convergence of the dynamical model of route choice. As various data collection and processing techniques are developed, a combination of dynamic models of route choice and traffic flow will rerplace more traditional mesoscopic traffic assignment models that are inadequate to depict drivers behavior in the $\mathrm{CV}$ environment. This study has limitations because of anticipating and combining emerging technologies that are not yet mature. If a discrete-event network simulator, such as ns-3, GloMoSim, or NetSim, handled the vehicle data in this study, reliability would be guaranteed. However, it is difficult to transform the network simulator equation appropriately because of their specification; therefore, each CV vehicle is applied to the ACC algorithm in this study. Use of experimental data will provide more realistic results in the foreseeable future. However, investigation of practical performance even in small networks can provide some unexpected results. Thus, we have to improve this stability formula and know how to evaluate the road condition in CV environment exactly. If we obtain real vehicle trajectory data, it will be good to fit a simulation model by modifying car-following theories. These results will provide a good foundation for further investigation and development of CV environments and related technologies. 


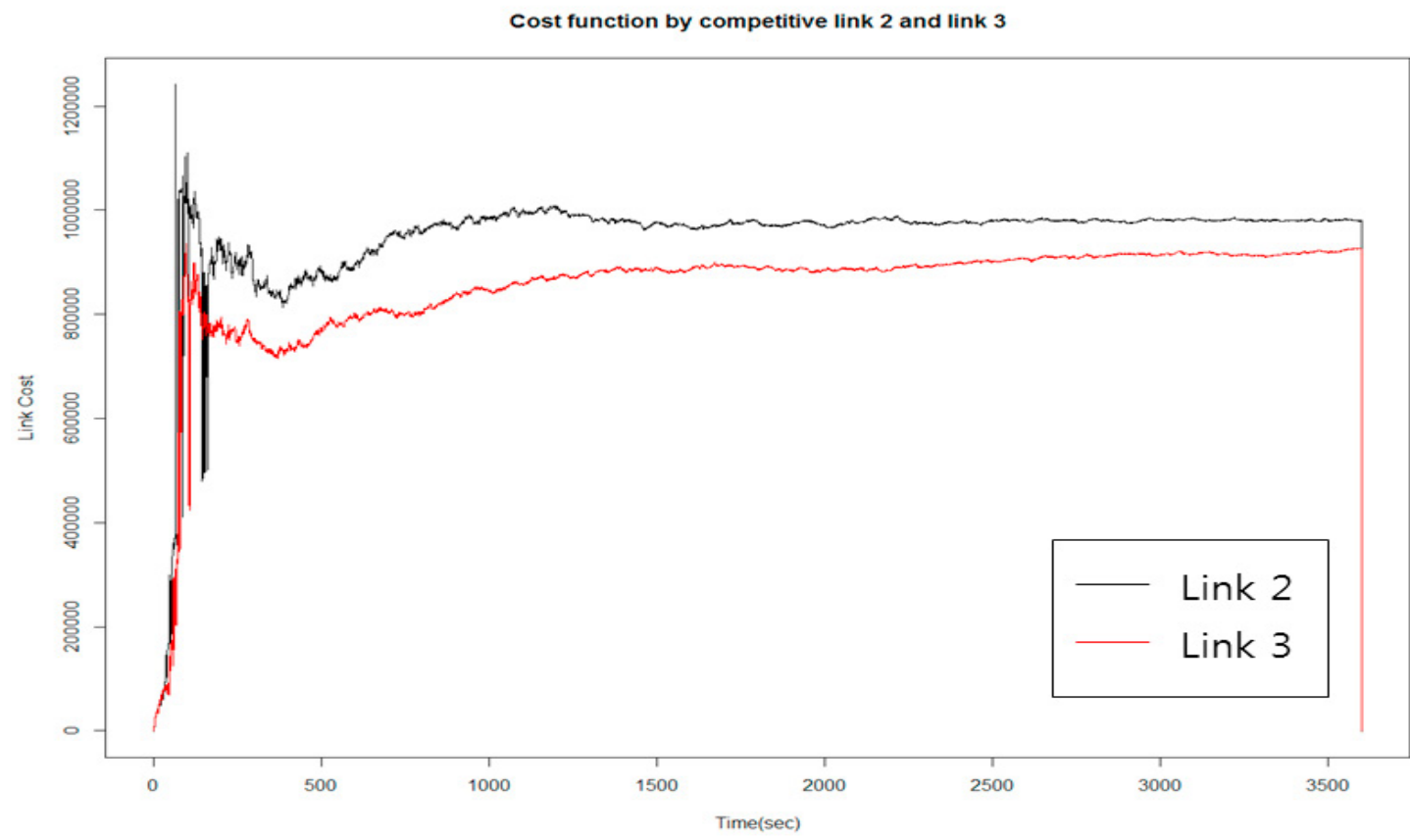

Fig. 9. Case 1 : Difference of cost by MPR $(10 \%, 30 \%, 80 \%)$

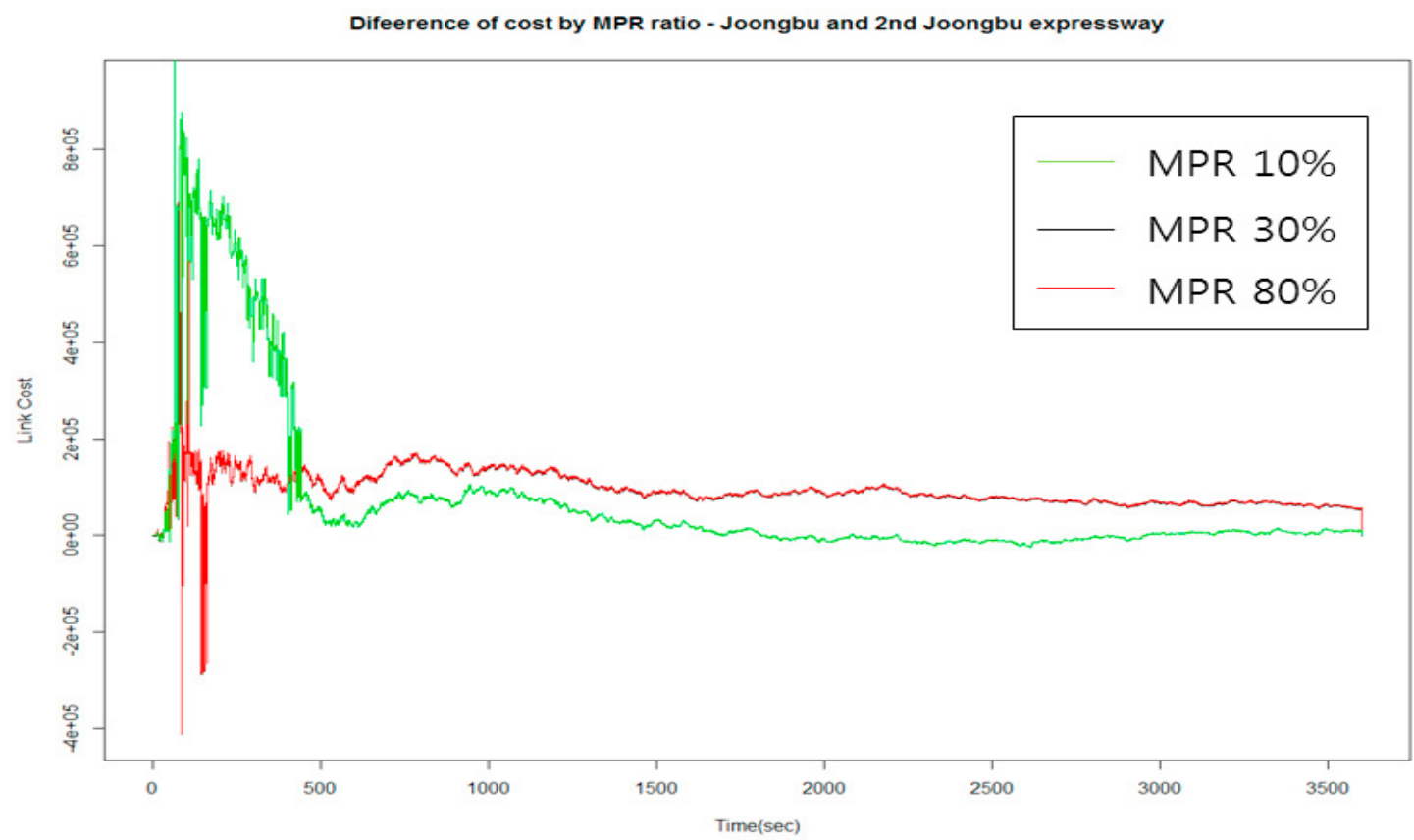

Fig. 10. Case 1 : Difference of cost by MPR $(10 \%, 30 \%, 80 \%)$

\section{Acknowledgements}

This work was supported by the National Research Foundation of Korea (NRF) grant funded by the Korean government (MSIP: Ministry of Science, ICT \& Future Planning) (No. NRF-2015R1A2A2A04005646). 


\section{Appendix A. Variational inequalities problem by using geometric (matrix) expression}

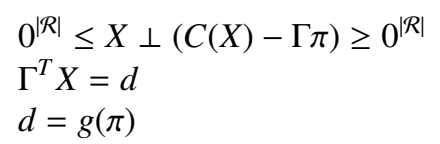

Another formulation involves the origin-destination (link) flows:

$$
\begin{aligned}
& 0^{|\mathcal{L}|} \leq w_{k} \perp\left(t(v)-E^{T} \pi_{k}\right) \geq 0^{|\mathcal{L}|}, \quad k \in C \\
& E w_{k}=i_{k} g_{k}\left(\left[i_{k}^{T} \pi_{k}\right]_{(x \in C)}\right), \quad k \in C \\
& \pi=\left(\pi_{p q}\right)_{(p, q) \in C}=\left(i_{k}^{T} \pi_{k}\right)_{k \in C} ;
\end{aligned}
$$

Then, $h \in D \cap S$ is a Wardrop equilibrium if and only if $T h=h$.

Linear program-solving variational inequality problem is presented by Lee (1995) as

$$
\begin{aligned}
& \min _{y \in X} t(x)^{T} y \\
& \left.\operatorname{gap}(\bar{h})=\max _{h, \bar{d} \in \hat{H}_{d}} t(\bar{h})^{T}(\overline{(} h)-h\right)=\sum_{k \in C} \max _{\left(h_{k}, \bar{d}_{k}\right) \in \hat{H}_{k, d}} t_{k}(\bar{h})^{T}\left(\bar{h}_{k}-h_{k}\right)
\end{aligned}
$$

To solve the variational inequality problem, a diagonalization algorithm is generally used. Before we adapt to this algorithm, transformation is needed. Given a function $t(v)$ in vector space with symmetric Jacobian, a minimization problem can be defined.

$$
v^{*}=\operatorname{argmin} \quad t(v)=\int_{0}^{v} t(v) d t
$$

$v \in S$ where $S$ is a nonempty, compact and convex set.

It is equal to the variational inequalities. Starting from a feasible point $v_{0} S$ obtained by all-or-nothing traffic assignment process, solve a succession of variational inequalities defined by functions with diagonal Jacobians represented by equations. In particular, at a point $v^{*} \in S$, the ith component function $t_{i}(v)$ of the function $t(v)$ can be approximated by a function $t_{i}^{*}\left(v_{i}\right)$ obtained by fixing all the other components of $v$ at their values $v_{j}^{*}$ (i.e., by diagonalizing the Jacobian):

$$
t_{i}\left(x_{1}, \ldots, x_{i-1}, x_{i}, x_{i+1}, \ldots\right) \cong t_{i}^{*}\left(x_{1}^{*}, \ldots, x_{i-1}^{*}, x_{i}, x_{i+1}^{*}, \ldots\right)=t_{i}^{*}\left(x_{i}^{*}\right), \forall i
$$

Thus, the variational inequality can be approximated by a variational inequality defined by a function of $t_{i}^{*}\left(x_{i}^{*}\right)$ with diagonal Jacobian values. The solution of approximate inequality can be obtained by solving the equivalent minimization problem, with one of the algorithms described in

$$
t_{i}\left(x_{1}, \ldots, x_{i-1}, x_{i}, x_{i+1}, \ldots\right) \cong t_{i}^{*}\left(x_{1}^{*}, \ldots, x_{i-1}^{*}, x_{i}, x_{i+1}^{*}, \ldots\right)=t_{i}^{*}\left(x_{i}^{*}\right), \forall i
$$

Therefore, our objective function is as follows :

$$
\begin{aligned}
& \min _{y \in X} t(x)^{T} y \\
& \operatorname{gap}(\bar{h})=\max _{h, \bar{d} \in \hat{H}_{d}} t(\bar{h})^{T}(\overline{(}(h)-h)=\sum_{k \in C} \max _{\left(h_{k}, \bar{d}_{k}\right) \in \hat{H}_{k, d}} t_{k}(\bar{h})^{T}\left(\bar{h}_{k}-h_{k}\right)
\end{aligned}
$$

\section{Appendix B. Stable criterion of Gipps model}

Acceleration function of the Gipps model has three variables and derivatives, and the partial derivatives of these variables are given by the following formula:

$$
v_{e}^{\prime}(s)=\frac{1}{T}, a_{s}=\frac{b}{T\left(b T+v_{e}\right)}, a_{v}=-\frac{1}{T}, a_{v l}=\frac{v_{e}}{T\left(b T+v_{e}\right)}
$$

This results in the following criterion for string stability:

$$
v_{e}^{\prime}\left(s_{e}\right) \leq \frac{1}{2}\left(a_{v l}-a_{v}\right)
$$




\section{References}

Allsop, R.E., 1974. Some possibilities for using traffic control to influence trip distribution and route choice. In: Buckley, D.J. (Ed.), Proceedings of the 6th International Symposium on Transportation and Traffic Theory, Sydney. Elsevier, pp. 345374.

Bauza, R., \& Gozalvez, J., 2013. Traffic congestion detection in large-scale scenarios using vehicle-to-vehicle communications. Journal of Network and Computer Applications, 36(5), 1295-1307.

Becker, S., Bork, M., Dorisen, H. T., Geduld, G., Hofmann, O., Naab, K., \& Noecker, G., 1994. Summary of experiences with autonomous Intelligent Cruise Control (AICC). Part 2: results and conclusions. Proceedings 1st World Congress on Intelligent Vehicle Systems. (pp. 18361843).

Cascetta, E., 2009. Transportation systems analysis: models and applications (Vol. 29). Springer Science \& Business Media.

Gartner, N.H., 1976. Area traffic control and network equilibrium. In: Florian, M. (Ed.), Traffic Equilibrium Methods, Lecture Notes in Economics and Mathematical Systems, vol. 118. Springer Verlag, pp. 274297.

Naus, G., Vugts, R., Ploeg, J., Van de Molengraft, M., \& Steinbuch, M., 2009. Towards on-the-road implementation of cooperative adaptive cruise control. Proc. 16th World Congr. Exhib. Intell. Transp. Syst. Serv.

Ghali, M. O., \& Smith, M. J., 1995. A model for the dynamic system optimum traffic assignment problem. Transportation Research Part B: Methodological,29(3), 155-170.

Gipps, P. G., 1981. A behavioural car-following model for computer simulation. Transportation Research Part B: Methodological, 15(2), 105-111.

He, X., Guo, X., \& Liu, H. X., 2010. A link-based day-to-day traffic assignment model. Transportation Research Part B: Methodological, 44(4), 597-608.

Hoedemaeker, M., \& Brookhuis, K. A., 1998. Behavioural adaptation to driving with an adaptive cruise control (ACC). Transportation Research Part F: Traffic Psychology and Behaviour, 1(2), 95-106.

Hu, J., Park, B. B., \& Lee, Y. J., 2015. Coordinated transit signal priority supporting transit progression under Connected Vehicle Technology. Transportation Research Part C: Emerging Technologies, 55, 393-408.

Jia, D., \& Ngoduy, D., 2016. Enhanced cooperative car-following traffic model with the combination of V2V and V2I communication. Transportation Research Part B: Methodological, 90, 172-191.

Knorr, F., Baselt, D., Schreckenberg, M., \& Mauve, M., 2012. Reducing traffic jams via VANETs. IEEE Transactions on Vehicular Technology, 61(8), 3490-3498.

Lee, S., 1995. Mathematical programming algorithms for equilibrium road traffic assignment (Doctoral dissertation, University of London).

Liu, R., \& Smith, M., 2015. Route choice and traffic signal control: A study of the stability and instability of a new dynamical model of route choice and traffic signal control. Transportation Research Part B: Methodological, 77, 123-145.

Lyapunov, A.M., 1907. Probleme general de la stabilite de mouvement. Ann. Fac. Sci. Toulouse 9, 203474 (Reprinted in Ann. Math. Stud. No 17 (Princeton University Press), 1949).

Mahmassani, H. S., 2016. 50th Anniversary Invited ArticleAutonomous Vehicles and Connected Vehicle Systems: Flow and Operations Considerations. Transportation Science, 50(4), 1140-1162.

Marcotte, P., \& Patriksson, M., 2007. Traffic equilibrium. Handbooks in Operations Research and Management Science, 14, $623-713$.

Meneguzzer, C., 1995. An equilibrium route choice model with explicit treatment of the effect of intersections. Transportation Research Part B: Methodological, 29(5), 329-356.

Milanes, V., Shladover, S. E., Spring, J., Nowakowski, C., Kawazoe, H., \& Nakamura, M., 2014. Cooperative adaptive cruise control in real traffic situations. IEEE Transactions on Intelligent Transportation Systems, 15(1), 296-305.

Ran, B \& Boyce D., 1996. Modeling dynamic transportation networks.

Sheffi, Y., 1985. Urban transportation networks.

Smith, M.J., 1979a. The existence, uniqueness and stability of traffic equilibria. Transp. Res. 13B, 295304.

Smith, M.J., 1979b. Traffic control and route-choice; a simple example. Transp. Res. 13B, 289294.

Smith, M.J., 1984a. The stability of a dynamic model of traffic assignment an application of a method of Lyapunov. Transportation Science 18 (3), 245252.

Smith, M., 2015. Traffic signal control and route choice: A new assignment and control model which designs signal timings. Transportation Research Part C: Emerging Technologies, 58, 451-473.

Swaroop, D., \& Hedrick, J. K., 1996. String stability of interconnected systems. IEEE transactions on automatic control, 41(3), $349-357$.

Swaroop, D. V. A. H. G., 1997. String stability of interconnected systems: An application to platooning in automated highway systems. California Partners for Advanced Transit and Highways (PATH).

Talebpour, A., \& Mahmassani, H. S., 2016. Influence of connected and autonomous vehicles on traffic flow stability and throughput. Transportation Research Part C: Emerging Technologies, 71, 143-163.

Treiber, M., \& Kesting, A., 2013. Traffic flow dynamics. Traffic Flow Dynamics: Data, Models and Simulation, Springer-Verlag Berlin Heidelberg. 